\title{
Hacia un modelo de interfaces multimodales adaptables a los canales de aprendizaje en aplicaciones colaborativas como apoyo a la educación
}

\author{
Itzel Alessandra Reyes Flores ${ }^{1}$, Carmen Mezura-Godoy ${ }^{1}$, Gabriela Sánchez Morales ${ }^{2}$ \\ ${ }^{1}$ Universidad Veracruzana, Xalapa, Veracruz, \\ México \\ ${ }^{2}$ CONACYT- Universidad Veracruzana, Xalapa, Veracruz, \\ México
}

zS15019630@estudiantes.uv.mx, cmezura@uv.mx, gsanchezmo@conacyt.mx

\begin{abstract}
Resumen. Las interfaces multimodales permiten que la interacción humanocomputadora se dé en forma natural, y que la unión entre el mundo real con el digital sea totalmente transparente para el usuario. Actualmente, existen aplicaciones educativas que utilizan este tipo de interfaces; sin embargo, aún existen problemas con estos sistemas, por ejemplo, no consideran el trabajo en equipo, la adaptación al usuario es pobre, y existen pocos modelos que apoyen su desarrollo. Por tal motivo en este trabajo se muestra una versión preliminar de un modelo que considera las interfaces multimodales en aplicaciones colaborativas como apoyo en la educación, tomando en cuenta la adaptación al contexto como son los canales de aprendizaje de los usuarios, el ruido ambiental y los dispositivos en uso. Posteriormente se muestra una arquitectura para dar un panorama general de cómo se llevará a cabo la adaptación mediante mecanismos de inferencia.
\end{abstract}

Palabras clave: Interfaces multimodales, aplicaciones educativas, aplicaciones colaborativas, canales de aprendizaje, mecanismos de inferencia.

\section{Towards a Model of Adaptable Multimodal Interfaces Considering Learning Channels to support education}

\begin{abstract}
Multimodal interfaces allow a smooth human-computer interaction, and connection between real and digital worlds to be entirely transparent to the user. Most current educational applications using this kind of interfaces does not support collaboration and they are not adaptable. Furthermore, few models have been developed for development of these applications. In this paper, we propose a preliminary model of multimodal interfaces for educational collaborative applications. Moreover, a corresponding architecture is also proposed in order to give a general overview on how adaptation is supported by inference techniques.
\end{abstract}


Keywords: Multimodal interfaces, educational applications, groupware, learning channels, inference mechanisms.

\section{Introducción}

La investigación y el desarrollo de Interfaces de Usuario Multimodales (IUM) ha ido creciendo en los últimos años por la necesidad de tener sistemas que interactúen con el usuario de forma natural y transparente. Las IUM han demostrado tener muchas ventajas comparadas con interfaces mono modales, ya que las primeras facilitan el acceso a la información y a la funcionalidad del sistema a través de una gama de diferentes canales de entrada y de salida [1], evitan los errores, proveen robustez a la interfaz, ayudan al usuario a corregir errores o recuperarse de ellos más fácilmente, y añaden métodos de comunicación alternativos a diferentes situaciones y ambientes [2].

Dentro de las aplicaciones que consideran IUM, se encuentran, NUILAC, que posibilita interactuar con un Learning Managment System como Moodle [3]; PSYBENCH permite que los usuarios geográficamente distantes puedan colaborar en un espacio de trabajo compartido utilizando interfaces tangibles [4], AIR Conferencing que mediante grabaciones de video y conversiones de voz a texto el usuario puede repetir lo que se perdió al estar ausente de la conferencia [5], y Thunderwire genera un espacio compartido entre usuarios conectados utilizando únicamente audio [6].

Las IUM responden a la entrada de más de una modalidad (entrada táctil, reconocimiento de voz, salida audiovisual de voz, y otros), utilizando diferentes dispositivos de entrada y salida de la computadora, haciendo uso de diferentes canales sensoriales [7]. Por otro lado, se dice que la modalidad está estrechamente unida con los sentidos humanos. Raisamo [8] distingue tres modalidades (Visual, Auditiva, y Táctil) que están relacionadas con los canales de aprendizaje.

Los canales de aprendizaje son el conjunto de estrategias, métodos y tendencias concretas que se utilizan cuando se quiere aprender algo [9]. De acuerdo a [11], toda persona percibe la información a través de canales de aprendizaje, es decir, por medio de los ojos (canal visual), de los oídos (canal auditivo) y de sensaciones del cuerpo (canal kinestésico). Sin embargo, son muy pocas las aplicaciones que consideran esta característica para beneficiar la interacción del usuario con su interfaz, y en menor medida consideran la colaboración grupal. Dicha colaboración es uno de los objetivos principales de las aplicaciones colaborativas.

Las aplicaciones colaborativas o groupware, como normalmente se les llama en la Computer-Supported Cooperative Work, son aplicaciones que apoyan el trabajo en equipo [1], cuyo objetivo es ayudar a los grupos en la comunicación, la colaboración y la coordinación de sus actividades, trabajando en entornos comunes y virtuales [12]. Este tipo de aplicaciones a menudo son utilizadas en contextos educativos, permitiendo la participación activa y la interacción entre estudiantes y profesores que comparten sus ideas e información para lograr un objetivo. Google, por ejemplo, pone a disposición de los centros educativos de forma gratuita Google Apps para educación, que es un conjunto de herramientas como correo Gmail, Google Calendar, Google Drive, entre otros. Por otro lado, se han desarrollado aplicaciones como Mindmeister, que permite la colaboración entre estudiantes en línea para la elaboración colaborativa de mapas mentales que se pueden editar sucesivamente [13]. 
Aun cuando ya existe una gran cantidad de aplicaciones colaborativas que apoyan a la educación, aún existen desafíos en la interacción de los estudiantes con estas aplicaciones que imposibilitan su aprovechamiento total; por ejemplo, mostrar la misma interfaz para todos los participantes sin considerar algo tan importante como son los canales de aprendizaje. Esto hace que la interacción se dé utilizando todos los sentidos de manera general, permitiendo así, la posibilidad de que el estudiante presente distracciones en el proceso de aprendizaje y colaboración.

Es importante destacar que actualmente existen aplicaciones colaborativas multimodales como son, AwarenessMaps, diseñado para compartir información entre los usuarios mediante una serie de imágenes con actividades recientes, y AudioSpace, una interfaz de audio que permite a los usuarios especificar contextos que les interesa [14]. Aun cuando estas aplicaciones consideran la multimodalidad la mayoría no se adaptan al contexto, es decir, están hechas sin considerar las situaciones en el ambiente externo que pueden afectar la interacción multimodal; por ejemplo, un usuario interactúa con un sistema mediante las modalidades de audio y reconocimiento de voz. Sin embargo, el sistema no considera que esta interacción puede afectar la concentración de otras personas al rededor; tampoco considera si el ambiente externo es muy ruidoso, o si los dispositivos de entrada y salida necesarios para la interacción están conectados y disponibles.

Dado el preámbulo anterior, en este trabajo se muestra una versión preliminar de un modelo que considera las interfaces multimodales en aplicaciones colaborativas como apoyo a la educación, tomando en cuenta los canales de aprendizaje, el ambiente externo y los dispositivos de entrada y salida. Este artículo se organiza de la siguiente manera, en la sección 2 se presentan los modelos, frameworks y arquitecturas importantes para este tema de investigación; en la sección 3, el modelo propuesto y en la sección 4 se muestra la arquitectura, los mecanismos de inferencia para la adaptación y un escenario para ejemplificar el uso de la arquitectura. Finalmente, en la sección 5 se presentan las conclusiones y el trabajo a futuro.

\section{Estado del arte}

Existen pocos modelos de interfaces multimodales que consideran contextos educativos, sin embargo, ninguno está enfocado en aplicaciones colaborativas, ni toman en cuenta los canales de aprendizaje del usuario. Por lo que a continuación se describen modelos, frameworks y arquitecturas que se han desarrollado hasta hoy, pero que contemplan cada una de estas características por separado.

Algunas investigaciones se han enfocado en diseñar frameworks y arquitecturas para el desarrollo de interfaces multimodales; por ejemplo, el trabajo de Flipplo et al. [15] provee un framework para el desarrollo rápido de estas interfaces, en las que los usuarios tienen la libertad de elegir y combinar la interacción con el sistema y así crear una forma más eficiente y agradable de trabajo. Dynamo [16] una arquitectura para diseñar interfaces multimodales adaptables a entornos generalizados, basados en servicios; esta arquitectura toma en cuenta la plataforma, el peso de los componentes y un gestor autónomo basado en modelos para construir y mantener la interacción multimodal en tiempo de ejecución. 
AMENITIES es una arquitectura centrada en el concepto de grupo basada en modelos de comportamiento y tareas para el análisis, diseño y desarrollo de sistemas cooperativos [17]. Y el modelo de Jun et al [18] para E-learning, basado en la tecnología que permite el trabajo en grupo, en el que se utilizan redes para mejorar la eficiencia del aprendizaje, la creación y el intercambio de conocimientos.

En la investigación y desarrollo de sistemas educativos también se han comenzado a desarrollar modelos. Uno de ellos es el modelo de Silva et al [19] basado en técnicas instruccionales y estilos de aprendizaje para el apoyo en la creación de Objetos de Aprendizaje (OA). Este modelo permite al profesor, a través del uso de una plataforma tecnológica, definir los objetivos de aprendizaje y los procesos cognitivos implicados. De igual forma el profesor define el estilo de aprendizaje de los estudiantes a quien va dirigido el $\mathrm{OA}$, el modelo evalúa 36 técnicas instruccionales; posteriormente, selecciona las más adecuadas a los procesos cognitivos y a los estilos de aprendizaje utilizando un modelo matemático.

Tabla 1. Modelos, frameworks y arquitecturas desarrolladas actualmente.

\begin{tabular}{|c|c|c|c|c|c|}
\hline Nombre & Enfoque & Colaborativo & Multimodal & $\begin{array}{l}\text { Adaptable } \\
\text { al usuario }\end{array}$ & $\begin{array}{l}\text { Aplicación } \\
\text { desarrollada }\end{array}$ \\
\hline $\begin{array}{l}\text { Framework de } \\
\text { Flipplo et al } \\
\text { (2003). }\end{array}$ & General & No & $\begin{array}{l}\text { Señalamiento } \\
\text { por } \\
\text { dispositivo y } \\
\text { Gaze }\end{array}$ & No & $\begin{array}{l}\text { Flatscape } \\
\text { (Aplicación } \\
\text { Colaborativa) }\end{array}$ \\
\hline $\begin{array}{l}\text { Arquitectura } \\
\text { Dynamo } \\
(2012) \text {. }\end{array}$ & General & No & $\begin{array}{l}\text { Señalamiento } \\
\text { con } \\
\text { dispositivo }\end{array}$ & No & $\mathrm{No}^{3}$ \\
\hline $\begin{array}{l}\text { Arquitectura } \\
\text { AMENITIES } \\
(2004) .\end{array}$ & General & $\mathrm{Si}$ & No & No & $\begin{array}{l}\text { Agenda } \\
\text { Colaborativa }\end{array}$ \\
\hline $\begin{array}{l}\text { Modelo de Jun } \\
\text { et al (2011). }\end{array}$ & Educativo & $\mathrm{Si}$ & No & No & No \\
\hline $\begin{array}{l}\text { Modelo de } \\
\text { Silva et al } \\
\text { (2012). }\end{array}$ & Educativo & No & No & $\begin{array}{l}\text { Estilos de } \\
\text { Aprendizaje, } \\
\text { Objetivos de } \\
\text { Aprendizaje. }\end{array}$ & $\begin{array}{l}\text { Plataforma } \\
\text { Tecnológica }\end{array}$ \\
\hline $\begin{array}{l}\text { Modelo de } \\
\text { Luna } \\
(2016) .\end{array}$ & Educativo & No & No & $\begin{array}{l}\text { Canales de } \\
\text { Aprendizaje, Estilo } \\
\text { de } \\
\text { Aprendizaje, } \\
\text { Motivación, } \\
\text { Cultura, Limitación, } \\
\text { Modelo mental, } \\
\text { Estrategia de } \\
\text { Aprendizaje. }\end{array}$ & $\mathrm{No}^{3}$ \\
\hline
\end{tabular}

\footnotetext{
${ }^{3}$ Se describen escenarios en lugar de desarrollarse alguna aplicación utilizando este modelo o arquitectura.
} 
Otro modelo que considera contextos educativos y canales de aprendizaje es el propuesto por Luna [11], para el desarrollo de aplicaciones interactivas en el que conceptualiza las características propias del usuario, estilos, canales de aprendizaje y su experiencia en el uso de sistemas educativos.

En la Tabla 1 se comparan los trabajos anteriormente mencionados, destacando principalmente su enfoque y el desarrollo de aplicaciones colaborativas; además si consideran la multimodalidad, al usuario y el desarrollo de alguna aplicación.

En la Tabla 1 se observa que dos de los seis trabajos toman en cuenta la multimodalidad, y con uno de ellos se desarrolló una aplicación colaborativa como caso de estudio. Sólo dos se enfocan en el desarrollo de aplicaciones colaborativas, y tres en el desarrollo de aplicaciones educativas que se adaptan al usuario; por ejemplo, consideran el estilo de aprendizaje, objetivos de aprendizaje, cultura etc.

Aun cuando estos trabajos de investigación consideran contextos educativos, aplicaciones colaborativas, interfaces multimodales y la adaptación del usuario, ninguno contempla unir estos elementos para enriquecer la interacción del usuario, como se propone en este trabajo. Es importante mencionar que el modelo que más se asemeja a esta propuesta es el de Luna [11], solo que este no considera las modalidades ni tampoco el trabajo colaborativo. Por tal motivo, se contempla la necesidad de crear un modelo que utilice interfaces multimodales adaptables a los canales de aprendizaje en aplicaciones colaborativas como apoyo a la educación.

\section{Propuesta del modelo}

En esta sección se describe una versión preliminar del modelo de interfaces multimodales adaptables a los canales de aprendizaje en aplicaciones colaborativas como apoyo a la educación. Este modelo considera ocho elementos: usuario, canal de aprendizaje, dispositivos de interacción, equipo heterogéneo, rol, actividad, ambiente externo, y aplicación colaborativa; y dos subelementos dentro de la actividad: tareas cooperativas y tareas colaborativas. En la Fig. 1, pueden observarse los elementos, subelementos y las relaciones entre ellos.

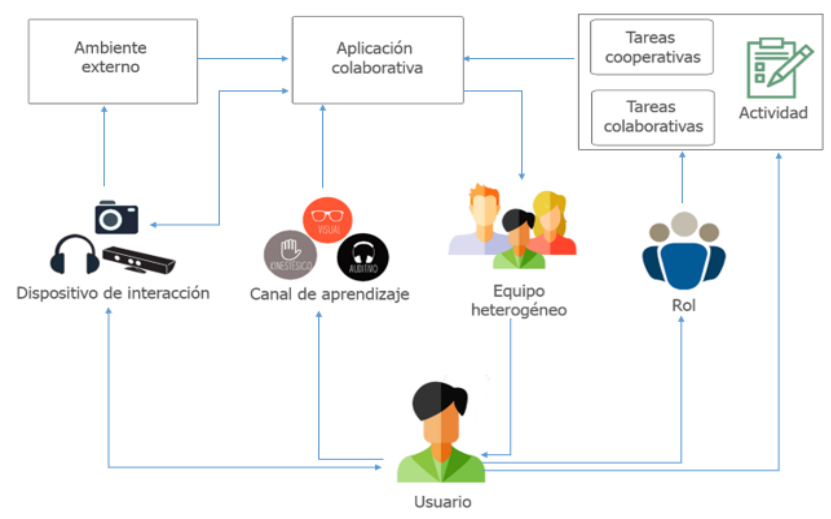

Fig. 1. Modelo de interfaces multimodales adaptables a los canales de aprendizaje en aplicaciones colaborativas como apoyo a la educación. 


\subsection{Elementos}

El elemento usuario es la persona física que utiliza la aplicación, ya sea el profesor o el alumno.

El canal de aprendizaje se refiere al modo en el que el usuario percibe la información. Usualmente predomina un solo canal en el alumno. Se consideran tres canales de aprendizaje: visual, auditivo y kinestésico.

Los dispositivos de interacción son los que permiten la comunicación entre el usuario con la aplicación. Éstos pueden ser de entrada, de salida o ambas.

El ambiente externo es la información que se obtiene de lo que pasa alrededor de la aplicación. Específicamente sobre los dispositivos conectados o desconectados a la computadora, la cantidad de personas que se encuentren alrededor del usuario y el ruido del ambiente.

Un equipo heterogéneo es un grupo de trabajo conformado por usuarios con diferentes canales de aprendizaje. En un caso ideal cada equipo tendrá un integrante de cada canal (visual, auditivo y kinestésico); de otra forma, se procurará formar equipos con al menos dos tipos de canales de aprendizaje diferentes.

Un rol es el papel que desarrolla el usuario dentro de la actividad colaborativa.

La actividad es el conjunto de operaciones que se llevan a cabo para cumplir un objetivo. Ésta consiste en la ejecución de ciertas tareas mediante la utilización de diversos recursos.

La aplicación colaborativa se refiere a los métodos que facilitan el trabajo en grupo, para este tema de investigación se quiere apoyar a los usuarios localizados en diferentes puntos geográficos a que trabajen en un objetivo en común. En la sección 4 se describe la arquitectura de éste elemento.

La actividad está conformada por tareas cooperativas y tareas colaborativas. En una tarea cooperativa los alumnos se dividen en sub tareas y cada quien se hará responsable de cumplir con su parte. En cambio, en la tarea colaborativa todos pueden trabajar en todas las sub tareas

\subsection{Relaciones}

Cada elemento del modelo está relacionado con otro. El usuario tiene un canal de aprendizaje, un dispositivo de interacción, un rol, una actividad y es parte de un equipo heterogéneo.

La aplicación colaborativa recibe como entrada el canal de aprendizaje que predomina en el usuario y mediante mecanismos de inferencia podrá asignar los equipos heterogéneos. Además, recibe la información del ambiente externo, y las tareas asignadas a cada integrante del equipo; esto, junto con el canal de aprendizaje, le permitirán activar, también mediante mecanismos de inferencia, la modalidad con la que va a interactuar el usuario con la interfaz, y los componentes con los que será conformada.

El rol del usuario intervendrá en la asignación de actividades. Cuando el usuario tiene un rol de profesor, este podrá asignar una actividad, y cuando sea un alumno será quien realice las tareas cooperativas o las tareas colaborativas en las que se dividió la actividad, asignándole a cada usuario una tarea y con ello un nuevo rol. 


\section{Propuesta de la arquitectura}

Con base en el modelo propuesto se describe una arquitectura de uno de sus elementos: aplicación colaborativa. En esta arquitectura se toman en cuenta cuatro elementos importantes: interfaz de usuario, adaptación, lógica del negocio y comunicación entre dispositivos. En la Fig. 2 se pueden identificar estos elementos y la relación que existe entre cada uno.

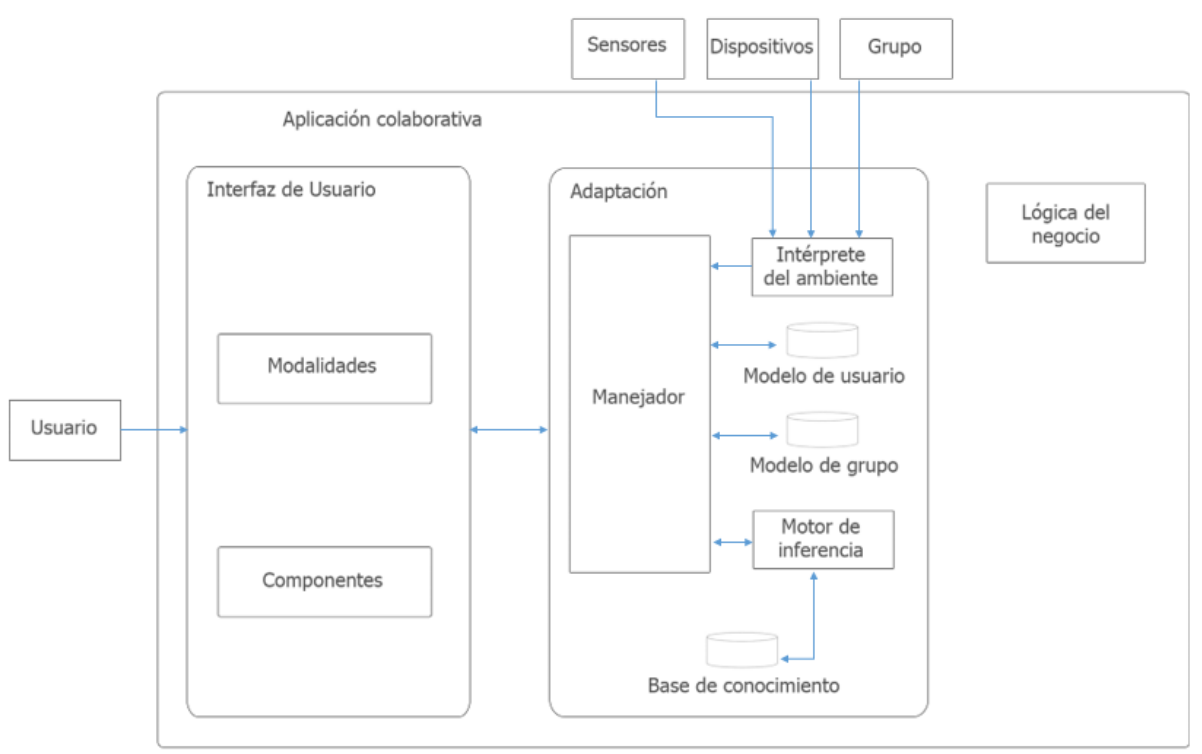

Fig. 2. Arquitectura de la aplicación colaborativa.

La interfaz de usuario es el medio de interacción entre el usuario y la aplicación. Por lo que la interfaz recibe como entrada el usuario que interactúa en ese momento con ésta. Dentro de la interfaz de usuario se consideran dos elementos; las modalidades de interacción, que significan el total de modalidades con el que el usuario podría interactuar con la aplicación. Éstas pueden ser reconocimiento de voz, audio, reconocimiento de la mirada, reconocimiento de gestos, entre otros. Así mismo se consideran los componentes que serán los elementos que conformarán la interfaz, ya sean botones, menús, barras y otros.

La adaptación le da a la aplicación, la capacidad de aprender y cambiar considerando la información que recibe del ambiente externo mediante los sensores, los dispositivos de interacción y un grupo determinado que se busque distribuir en equipos más pequeños de forma heterogénea. Dicha capacidad de aprender se realizará con mecanismos de inferencia. En específico la adaptación activará la modalidad con la que el usuario va a interactuar con la interfaz, y asignará los equipos heterogéneos de un grupo determinado.

La información obtenida por el ambiente externo es encapsulada por el intérprete del ambiente (IA) y si es necesario lo traduce a otros formatos; por ejemplo, si alguna función de la aplicación utiliza un sensor de ruido, pero éste da resultados de un tipo 
diferente al que recibe como parámetros la función, es el IA el que se encarga de la traducción.

Así mismo, el IA se comunica con el manejador. Éste manejador utiliza los datos del intérprete para saber las acciones a realizar (hacer consultas, llamar funciones, generar nuevos hechos, entre otros). Además, el manejador guarda y recupera información del modelo de usuario y/o del modelo de grupo. El modelo de usuario almacena un conjunto de datos, de manera estructurada, que utiliza la aplicación para respaldar información acerca del usuario que utiliza la aplicación en ese momento. Y el modelo de grupo almacena los datos del grupo determinado, del cual se requieren formar equipos heterogéneos.

Para obtener resultados acerca de la modalidad a activar o de los equipos heterogéneos que habrá que conformar, el manejador le envía a un motor de inferencia la información del modelo de usuario y/o del modelo de grupo. Éste motor de inferencia, a su vez consulta una base de conocimientos que contiene hechos que se consideran ciertos e incuestionables, y un conjunto de reglas conformadas por premisas o términos y una conclusión. El motor consulta la lista de hechos y verifica si con ellas se cumplen todos los requisitos o premisas de una o más reglas; si esto es cierto, entonces se verifica que a la conclusión que se llega está dentro de la lista de hechos, sino está, entonces se agrega como uno nuevo, así la aplicación aprende nuevo conocimiento hasta llegar a una conclusión final. En este caso, activar la modalidad con la que el usuario va a interactuar con la interfaz o la cantidad de equipos heterogéneos conformados y cómo estarán distribuidos para realizar la actividad colaborativa.

Así mismo, dentro de la aplicación colaborativa, se considera la lógica del negocio que es el núcleo funcional de la aplicación, es decir, debe contener todos los requerimientos necesarios dependiendo la aplicación colaborativa, por ejemplo, editor de textos, lluvia de ideas, entre otros. Además, se encarga de la lógica detrás de la interfaz de usuario; pueden ser, entradas y consultas de datos, procesamiento de información y entrega de resultados al usuario.

\subsection{Escenario}

En el siguiente escenario se considera los elementos de la arquitectura, por tal motivo se encontrarán dichos elementos a lo largo de la descripción, así como las entradas a la aplicación colaborativa.

En un salón de clases se imparte la materia de lectura y redacción (grupo) a 10 estudiantes de licenciatura en informática. Durante la clase el profesor les solicita a los alumnos que realicen una presentación (actividad) en diapositivas para exponer sobre las reglas gramaticales. Esta actividad será en equipo, lo cual el profesor lo indica en la aplicación colaborativa de apoyo. Entonces la aplicación recupera la información de los estudiantes (modelo de usuario) para sugerir como organizarlos en equipos (motor de inferencia). De los 10 estudiantes se encuentran 7 con un canal de aprendizaje visual, 2 con auditivo y 1 con kinestésico, información que la aplicación colaborativa previamente conoce, por lo que propone tres opciones: 5 equipos conformados por 2 integrantes, 2 equipos formados por 5 integrantes o 3 equipos formados con diferente número de estudiantes ( 2 equipos de 3 personas y 1 equipo de 4 personas). Por ejemplo, en la primera opción tiene 2 equipos integrados con un alumno visual y un auditivo, 1 
equipo integrado con un alumno kinestésico y un visual, y 2 equipos integrados con dos alumnos visuales respectivamente. En este caso el profesor selecciona la opción uno.

El profesor ingresa a la aplicación, asigna la actividad, y la divide en sub tareas cooperativas. Dependiendo el rol de cada integrante del equipo (equipo heterogéneo) la aplicación asignará una sub tarea a cada integrante, y de acuerdo a su canal de aprendizaje designará la modalidad de interacción y los componentes de la interfaz.

Cada uno de los integrantes del equipo tienen que cumplir con la actividad fuera de la clase, así que ellos prefieren hacerla en casa, por lo cual cada uno de ellos ingresa a la aplicación desde su computadora. Supongamos que el alumno Pedro, tiene un canal auditivo, como tarea le corresponde escribir los títulos y subtítulos de cada diapositiva, y revisar la información que el resto de sus compañeros haya escrito. Tiene conectados sus audífonos con micrófono integrado (dispositivos), y se encuentra sólo en una habitación sin ruido (sensores), por lo que la aplicación le ha asignado las modalidades de reconocimiento de voz y audio (motor de inferencia).

\section{Conclusiones y trabajo futuro}

En este artículo se propone una versión preliminar de un modelo y una arquitectura que consideran las interfaces multimodales en aplicaciones colaborativas como apoyo a la educación, tomando en cuenta la adaptación al usuario, en específico a sus canales de aprendizaje. Como se habló en la sección de estado del arte, existen trabajos relacionados con estas características. Cabe mencionar que de los seis trabajos identificados sólo tres de estos tienen un enfoque educativo, como lo hace también esta investigación.

De igual forma, sólo dos de estos trabajos relacionados proponen interfaces utilizando modalidades como, señalamiento por dispositivo y Gaze. A diferencia de estos trabajos, el modelo planteado en esta investigación toma en cuenta multimodalidades relacionadas con los canales de aprendizaje; reconocimiento de voz, salida de audio (canal auditivo), salida visual, señalamiento por dispositivo (canal visual), y reconocimiento de gestos (canal kinestésico).

Por otra parte, como resultado de la investigación de la literatura, se identificó una arquitectura y un modelo que sirven de referencia para el desarrollo de aplicaciones colaborativas. Sin embargo, estos trabajos no incluyen interfaces multimodales como lo propone este trabajo, y no se adaptan al usuario.

Así mismo, cabe mencionar que en el estado del arte se identificaron dos modelos que toman en cuenta la adaptación al usuario, considerando características propias como, el estilo de aprendizaje, los objetivos de aprendizaje, los canales de aprendizaje, la motivación, la cultura, la limitación, el modelo mental, y la estrategia de aprendizaje. Sin embargo, el modelo propuesto en este trabajo contempla la adaptación al usuario considerando los canales de aprendizaje, el ambiente externo en el que el usuario se encuentra, los dispositivos de entrada y salida conectados al momento de interactuar con la aplicación, y las actividades que le fueron asignadas para determinar la modalidad de interacción entre el usuario y la interfaz. En la arquitectura propuesta se presentan los mecanismos de inferencia para adaptar la interfaz respecto a la modalidad que se activará dado el contexto del usuario y del ambiente. Además, se propone otro mecanismo de inferencia para distribuir de manera heterogénea los equipos de trabajo 
que utilizarán la aplicación, con el fin de que exista, en el mejor de los casos, un integrante de cada canal de aprendizaje (visual, auditivo, kinestésico).

Dado a que este trabajo de investigación aún se encuentra en desarrollo, como trabajo futuro, se desarrollarán los mecanismos de inferencia para asignar grupos heterogéneos de trabajo, para adaptar la interfaz y las modalidades de interacción. El modelo y la arquitectura planteada se probarán mediante un caso de estudio que consistirá en desarrollar una aplicación colaborativa como apoyo a la educación que utilice interfaces multimodales para la interacción con el alumno y se adapte de acuerdo a su canal de aprendizaje; por ejemplo, activar modalidades de reconocimiento de voz y audio para un alumno con canal auditivo, y adaptar la interfaz de usuario con un lector de pantalla. Actualmente, dicha aplicación está en la fase de análisis de requerimientos, en la cual se están incluyendo alumnos de la licenciatura en Informática de la Universidad Veracruzana. Además, como otro de los trabajos a futuro es el enriquecimiento del modelo y arquitectura propuesta, con el fin de considerar los elementos faltantes o sobrantes que se detecten al desarrollar la aplicación colaborativa.

Agradecimientos. Agradecemos a CONACYT por todos los apoyos, en específico por la beca con número 590658, así como a la Universidad Veracruzana por el apoyo brindado para el desarrollo de esta investigación.

\section{Referencias}

1. Dix, A., Finlay, J., Abowd, G., Beale, R.: Human-Computer Interaction. Pearson Education Limited. pp. 366-370, 663-665 (1993)

2. Jaimes, A., Sebe, N.: Multimodal human-computer interaction: A survey. Computer vision and image understanding. Vol. 108, No. 1, pp. 116-134 (2007)

3. Vásquez, A., Duque, E.: Nui para la educación, eliminando la discriminación tecnológica en la búsqueda de la inclusión digital. Corporación Universitaria Americana (2013)

4. Brave, S., Ishii, H., Dahley, A.: Tangible Interfaces for Remote Collaboration and Communication. Proceedings of the 1998 ACM conference on Computer supported cooperative work, ACM, pp. 169-178 (1998)

5. Inkpen, K., Hegde, R., Junuzovic, S., Brooks, C., Tang, J.C.: AIR Conferencing: Accelerated Instant Replay for In-Meeting Multimodal Review. Proceedings of the 18th ACM international conference on Multimedia, ACM, pp. 663-666 (2010)

6. Ackerman, M., Starr, B., Hindus, D., Mainwaring, S.: Hanging on the 'Wire: A Field Study of an Audio-Only Media Space. ACM Transactions on Computer-Human Interaction, Vol. 4, No. 1, pp. 39-66 (1997)

7. Möller, S., Engelbrecht, K., Kühnel, C., Wechsung, I., Weiss, B.: A taxonomy of Quality of Service and Quality of Experience of Multimodal Human-Machine Interaction. Quality of Multimedia Experience, QoMEx, International Workshop on. IEEE, pp. 7-12 (2009)

8. Raisamo, R.: Multimodal Human-Computer Interaction: a constructive and empirical study. Tampereen yliopisto (1999)

9. Tomas, U.: Canales del Aprendizaje. El psicoasesor. http://elpsicoasesor.com/canales-delaprendizaje/ (2011)

10. Valdivia, J.: El conocimiento de los estilos de aprendizaje como medida de atención a la diversidad y sus implicaciones educativas en educación infantil. Revista digital enfoques educativos, Vol. 75, pp. 85-94 (2011)

11. Luna, J.: Modelo de interacciones para el desarrollo de aplicaciones interactivas en contextos educativos. Universidad Veracruzana (2016) 
12. Ellis, C.A., Gibbs, S.J., Rein, G.L.: Groupware some issues and experiences. Communications of the ACM, Vol. 34, No.1, pp. 38-58 (1991)

13. Abdul, J., Velarded, R.: Herramientas Web 2.0 para el Aprendizaje Colaborativo. http://remo.det.uvigo.es/solite/attachments/038_Web\%202.0.pdf (2009)

14. Gross, T.: Universal Access to Groupware with Multimodal Interfaces. Proc. of the second Int. Conf. on Universal Access in HCI: Inclusive Design in the Information Society, pp. 1108-1112 (2003)

15. Flippo, F., Krebs, A., Marsic, I.: A Framework for Rapid Development of Multimodal Interfaces. Proceedings of the 5th international conference on Multimodal interfaces, ACM, pp. 109-116 (2003)

16. Avouac, P.A., Lalanda, P., Nigay, L.: Adaptable multimodal interfaces in pervasive environments. Consumer Communications and Networking Conference. IEEE, pp. 544$548(2012)$

17. Garrido, J.L., Gea, M., Noguera, M., González, M., Ibáñez, J.A.: Una Propuesta Arquitectónica para el Desarrollo de Aplicaciones Colaborativas. Interacción, pp. 164-171 (2004)

18. Jun, Y., Suicheng, L., Rui, H.: The model of Networked Learning Environment for Elearning Based on Groupware Technology. Computer Science \& Education (ICCSE), 6th International Conference on, Vol. 8, No. 11, pp. 995-998 (2011)

19. Silva, A., Ponce, J.C., Villalpando, M.D.: Modelo para la Creación y Uso de Objetos de Aprendizaje, Basado en la Valoración de Técnicas Instruccionales. Conferencias LACLO, Vol. 3, No. 1 (2012) 